\title{
Temporal mesonic correlators at NLO for any quark mass
}

\author{
Y. Burnier ${ }^{* a}$ and M. Laine ${ }^{b}$ \\ ${ }^{a}$ Institute of Theoretical Physics, EPFL, CH-1015 Lausanne, Switzerland \\ ${ }^{b}$ Institute for Theoretical Physics, AEC, University of Bern, CH-3012 Bern, Switzerland \\ E-mail: yannis.burnier@epfl.ch, laineditp.unibe.ch
}

\begin{abstract}
We present NLO results for thermal imaginary-time correlators in the vector and scalar channels as a function of the quark mass. The range of quark masses for which a non-relativistic approximation works in the temperature range considered is estimated, and charm quarks turn out to be a borderline case. Comparing with simulation data from fine lattices, we find good agreement in the vector channel but a substantial discrepancy in the scalar one. An explanation for the discrepancy is suggested in terms of physics of the quark-antiquark threshold region. Perturbative predictions for the bottom scalar spectral function around the threshold are also briefly reviewed.
\end{abstract}

31 st International Symposium on Lattice Field Theory LATTICE 2013

July 29 - August 3, 2013

Mainz, Germany

\footnotetext{
*Speaker.
} 


\section{Motivation}

Massive quarks have long been considered as excellent probes for the physics of a quarkgluon plasma. Due to their heavy mass they could experience changes at finite temperature that are both theoretically tractable and experimentally identifiable. The fortunate existence of both charm and bottom quarks in an appropriate mass range implies that the quark mass can be considered a tunable parameter and that theoretical predictions may be interpolated or extrapolated as a function of the quark mass. The fates of "open" heavy-flavour D and B mesons on one hand, and "bound" charmonium and bottomonium systems on the other, capture a rich spectrum of interesting physics phenomena. Heavy quarks are also relatively easy to simulate on the lattice, even though care needs to be taken in view of possible discretization artefacts.

The present study is related to lattice measurements of two-point correlators of heavy scalar densities and vector currents at finite temperature. Ultimately, the goal is to use imaginary-time correlators measured on the lattice in order to constrain the corresponding spectral functions; the latter, in turn, describe open heavy flavour physics through a transport peak at small frequency, and quarkonium physics through a threshold region at large frequency. In the present investigation the main focus is on imaginary-time correlators, which we have recently computed up to next-toleading order (NLO) as a function of the quark mass $[1,2]$ and compared with quenched data from fine lattices [3].

\section{Charm quark imaginary-time correlators}

The vector and scalar correlators are defined in continuum as

$$
\begin{aligned}
G_{i i}(\tau) & \equiv \sum_{i=1}^{3} \int_{\mathbf{x}}\left\langle\left(\bar{\psi} \gamma_{i} \psi\right)(\tau, \mathbf{x})\left(\bar{\psi} \gamma_{i} \psi\right)(0, \mathbf{0})\right\rangle_{T}, \\
G_{\mathrm{S}}(\tau) & \equiv M_{\mathrm{B}}^{2} \int_{\mathbf{x}}\langle(\bar{\psi} \psi)(\tau, \mathbf{x})(\bar{\psi} \psi)(0, \mathbf{0})\rangle_{T},
\end{aligned}
$$

where $M_{\mathrm{B}}$ is the bare quark mass and $0<\tau<1 / T$. As is clear from the definitions, the scalar correlator is more sensitive to the quark mass than the vector one. (Without the bare quark mass in the definition, the scalar correlator would not be renormalizable even at NLO, and it would lose its connection to the QCD Lagrangian.)

Physically, the charm quark vector correlator is related to an in-medium $J / \psi$ contribution to the thermal dilepton production rate, as well as to the charm quark diffusion coefficient and kinetic equilibration rate. The scalar density operator represents the quark contribution to the trace of the energy-momentum tensor and is hence related to the bulk viscosity of the quark-gluon plasma as well as to the charm quark chemical equilibration rate. In addition, the scalar spectral function around threshold is believed to describe $P$-wave charmonium states.

The lattice and NLO results for the two correlators are compared in fig. 1. The results are normalized to massless "free" correlators,

$$
\begin{aligned}
& G_{i i}^{\text {free }}(\tau) \equiv 2 N_{\mathrm{c}} T^{3}\left[\pi(1-2 \tau T) \frac{1+\cos ^{2}(2 \pi \tau T)}{\sin ^{3}(2 \pi \tau T)}+\frac{2 \cos (2 \pi \tau T)}{\sin ^{2}(2 \pi \tau T)}+\frac{1}{6}\right], \\
& G_{\mathrm{S}}^{\text {free }}(\tau) \equiv N_{\mathrm{c}} T^{3} m_{\tau}^{2}\left[\pi(1-2 \tau T) \frac{1+\cos ^{2}(2 \pi \tau T)}{\sin ^{3}(2 \pi \tau T)}+\frac{2 \cos (2 \pi \tau T)}{\sin ^{2}(2 \pi \tau T)}\right],
\end{aligned}
$$



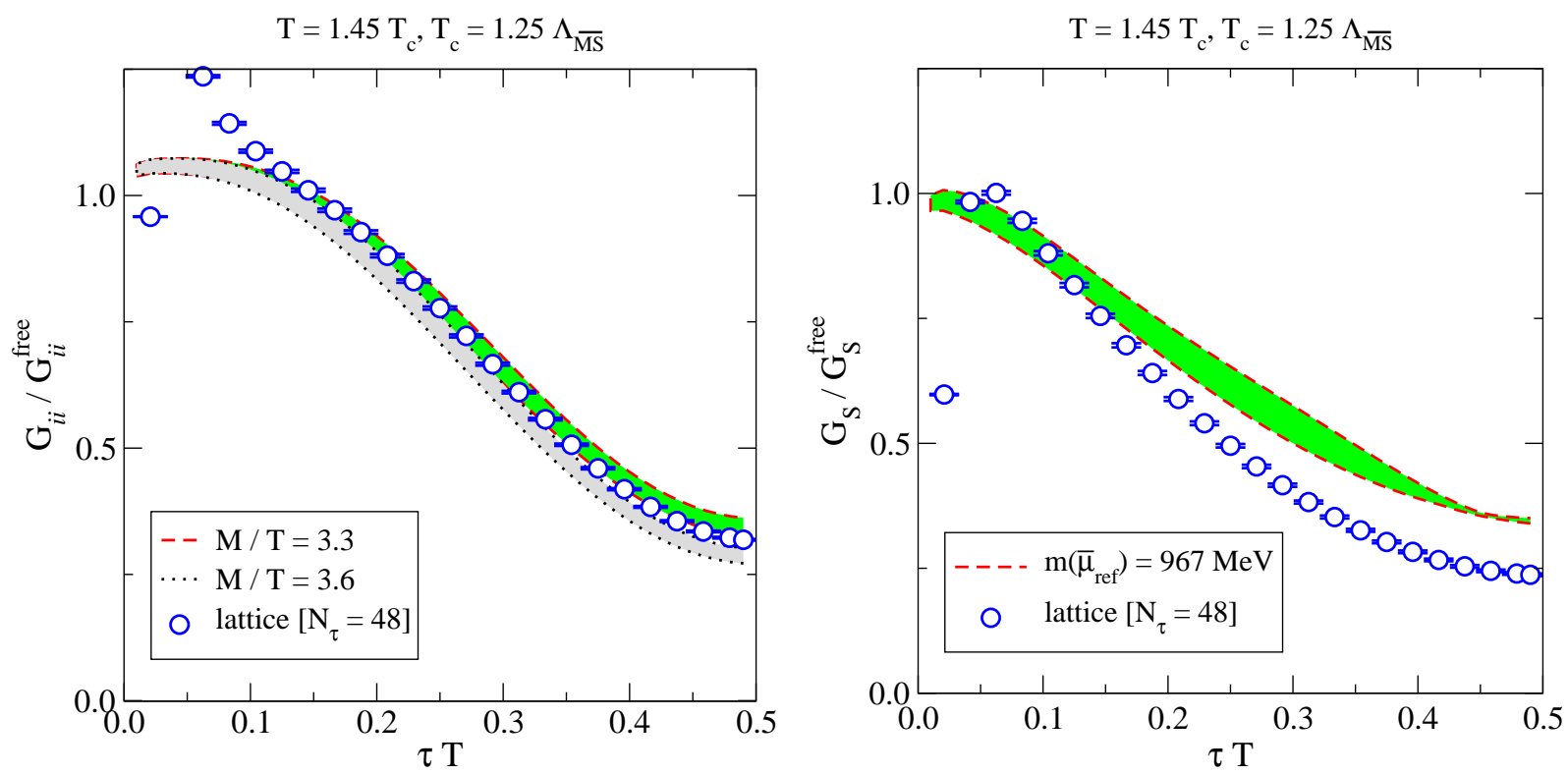

Figure 1: Left: The spatial part of the vector correlator, eq. (2.1), normalized to eq. (2.3), compared with lattice data from ref. [3]. The pole masses $M / T=3.3,3.6$ are chosen from a perturbative estimate and from an optimal agreement of quark number susceptibility, respectively [1]. Right: The scalar correlator, eq. (2.2), normalized to eq. (2.4), compared with lattice data from ref. [3]. The $\overline{\mathrm{MS}}$ mass $m\left(\bar{\mu}_{\text {ref }}\right)=967 \mathrm{MeV}$, with $\bar{\mu}_{\text {ref }}=2 \mathrm{GeV}$ [4], corresponds to the value $m_{c}\left(m_{c}\right)=1.094(1) \mathrm{GeV}$ cited in ref. [3]. (In the scalar channel the pole mass scheme shows questionable convergence already at NLO [2].)

where we have defined $m_{\tau}^{2} \equiv m^{2}\left(\bar{\mu}_{\mathrm{ref}}\right)\left\{\ln \left[\frac{\bar{\mu}_{\mathrm{ref}}}{\Lambda_{\overline{\mathrm{MS}}}}\right] / \ln \left[\frac{\beta e^{\frac{1}{12}-\gamma_{\mathrm{E}}}}{\tau(\beta-\tau) \Lambda_{\overline{\mathrm{MS}}}}\right]\right\}^{\frac{18 C_{\mathrm{F}}}{11 N_{\mathrm{c}}-4 T_{\mathrm{F}}}}, \bar{\mu}_{\text {ref }} \equiv 2 \mathrm{GeV}[4], \beta \equiv 1 / T$, $C_{\mathrm{F}} \equiv\left(N_{\mathrm{c}}^{2}-1\right) /\left(2 N_{\mathrm{c}}\right)$, and $T_{\mathrm{F}} \equiv N_{\mathrm{f}} / 2$. Moreover $m(\bar{\mu})$ is the $\overline{\mathrm{MS}}$ scheme quark mass.

It can be observed from fig. 1 that in the vector channel the results agree well (apart from discretization artefacts at small $\tau$ ). In contrast, in the scalar channel a clear discrepancy is visible. In the following, we concentrate on understanding what is going on in the scalar correlator.

\section{Validity of the non-relativistic approximation}

In order to investigate possible reasons for the discrepancy in fig. 1(right), it is useful to view the imaginary-time correlator as originating from an underlying spectral function $\rho_{\mathrm{S}}$,

$$
G_{\mathrm{S}}(\tau)=\int_{0}^{\infty} \frac{\mathrm{d} \omega}{\pi} \rho_{\mathrm{S}}(\omega) \frac{\cosh \left[\left(\frac{\beta}{2}-\tau\right) \omega\right]}{\sinh \left(\frac{\beta \omega}{2}\right)} .
$$

The spectral function represents the cut (imaginary part) of a two-point correlator in momentum space. Surprisingly, spectral functions are not particularly well studied in the presence of a quark mass: even in vacuum, the result is known analytically only up to NLO (cf. ref. [5] and references therein; numerical estimates exist also at higher orders, cf. ref. [6]). For $m \neq 0$ NLO thermal corrections to $\rho_{\mathrm{S}}$ have been computed only for the "non-relativistic" regime $m \gg \pi T$ [7]. (This is peculiar since the generalization of massless zero-momentum NLO spectral functions to a finite mass should be less complicated than to a finite momentum [8].) 


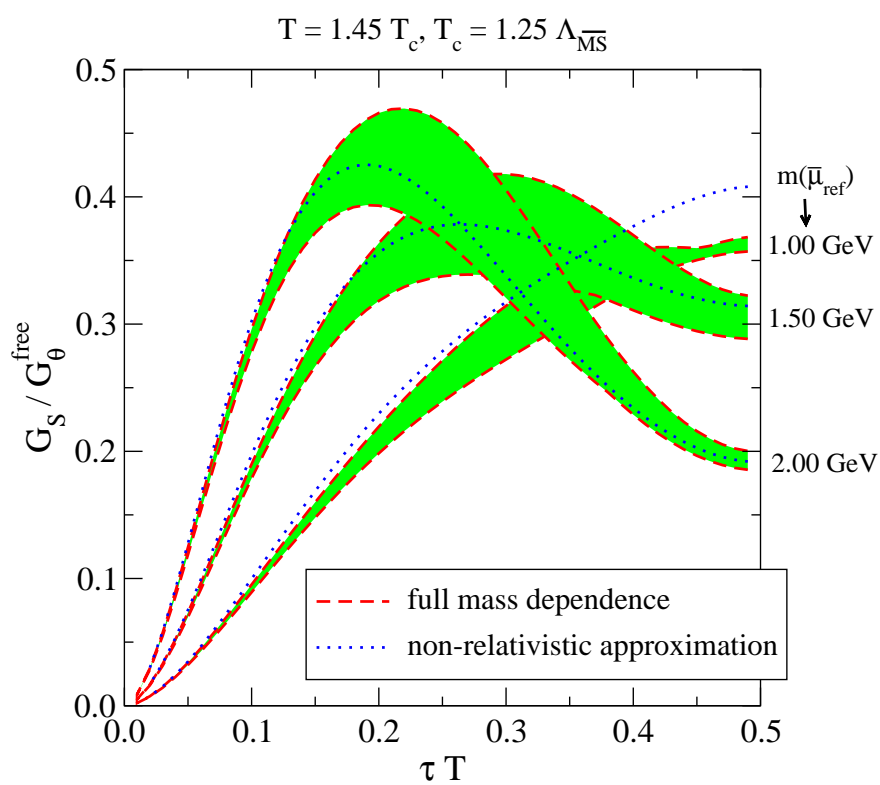

Figure 2: Comparison of NLO scalar correlators with full mass dependence (bands), against results based on a non-relativistic approximation of the NLO spectral function at large $\omega$ [7] together with a constant contribution from the transport peak at small $\omega$ [2] (dotted lines), normalized to eq. (3.2). The non-relativistic approximation is accurate for $m\left(\bar{\mu}_{\text {ref }}\right) \gtrsim 1.5 \mathrm{GeV}$ at this temperature, which is above the physical value $m_{c}\left(\bar{\mu}_{\text {ref }}\right)=1.275(25) \mathrm{GeV}$ [4]. However, even for $m\left(\bar{\mu}_{\text {ref }}\right)=1.0 \mathrm{GeV}$, its breakdown is not catastrophic.

It is useful to start by asking how well the non-relativistic results of ref. [7] compare with the numerically determined imaginary-time correlators computed in ref. [2], where no approximation was made with respect to the quark mass. For this purpose, the contribution of the transport peak, which was not addressed in ref. [7], needs to be added. Within NLO perturbation theory the transport peak yields an exactly $\tau$-independent contribution, given in eqs. (4.8), (4.10), (4.13) of ref. [2]. We sum this to the contribution from the spectral function, and normalize the results to a purely gluonic scalar correlator, viz.

$$
\frac{G_{\theta}^{\text {free }}(\tau)}{2 N_{\mathrm{c}} C_{\mathrm{F}} T^{5}} \equiv\left(8 \pi c_{\theta} g_{\tau}^{2}\right)^{2}\left[\pi(1-2 \tau T) \frac{2 \cos (2 \pi \tau T)+\cos ^{3}(2 \pi \tau T)}{\sin ^{5}(2 \pi \tau T)}+\frac{1+2 \cos ^{2}(2 \pi \tau T)}{\sin ^{4}(2 \pi \tau T)}\right]
$$

where $g_{\tau}^{2} \equiv 24 \pi^{2} /\left\{\left(11 N_{\mathrm{c}}-4 T_{\mathrm{F}}\right) \ln \left[\frac{\beta e^{\frac{14}{33}-\gamma_{\mathrm{E}}}}{\tau(\beta-\tau) \Lambda_{\overline{\mathrm{MS}}}}\right]\right\}, c_{\theta} \equiv-b_{0} / 2-b_{1} g_{\tau}^{2} / 4$, and $b_{0}, b_{1}$ are coefficients of the QCD $\beta_{g}$-function. The justification for this normalization is that it is mass-independent and conveniently magnifies the interesting large- $\tau$ regime.

The result of the comparison is shown in fig. 2. We observe that the non-relativistic approximation is accurate for $m\left(\bar{\mu}_{\text {ref }}\right)=2 \mathrm{GeV}$, whereas for $m\left(\bar{\mu}_{\text {ref }}\right)=1 \mathrm{GeV}$ (which is close to $m\left(\bar{\mu}_{\text {ref }}\right)=$ $967 \mathrm{MeV}$ simulated in ref. [3]) a discrepancy is visible. The physical case, with $m_{c}\left(\bar{\mu}_{\text {ref }}\right)=$ $1.275(25) \mathrm{GeV}$ [4], lies in between, however surely not deep in the non-relativistic regime. This is interesting in its own right, because if the charm quarks are not really exponentially suppressed, then the question of their partial chemical equilibration may be raised [9]. 

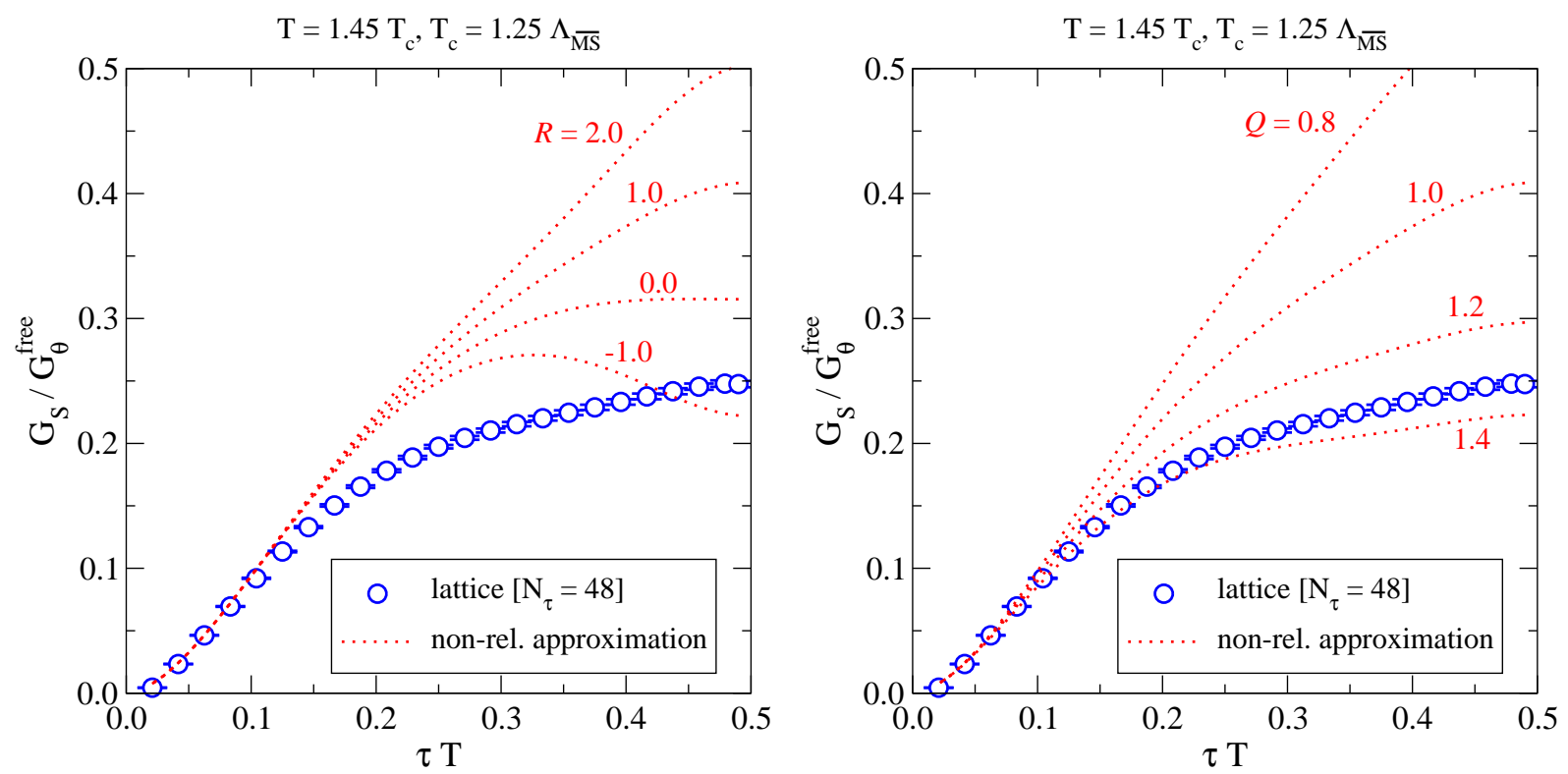

Figure 3: Left: Modification of the scalar correlator if the contribution from the transport peak is multiplied by a factor $R$, cf. eq. (4.1). It is seen that even a large deviation from $R=1$ does not help. Right: Modification of the scalar correlator if the threshold location is shifted left or right by a multiplicative factor $Q$, cf. eq. (4.3). A substantial improvement can be observed. Both plots are based on a non-relativistic approximation in the regime $\omega>m\left(\bar{\mu}_{\text {ref }}\right)=967 \mathrm{MeV}$, omitting terms suppressed by $e^{-m\left(\bar{\mu}_{\text {ref }}\right) / T}$.

\section{Explaining the discrepancy in the scalar channel}

Moving on, we have carried out two tests in order to probe the origins of the discrepancy seen in fig. 1(right). The first test concerns the contribution of the transport peak. As mentioned, this yields a constant contribution within NLO perturbation theory. We have tested how changing the amplitude of the constant by a factor $R$ changes the result:

$$
\left.\left.G_{\mathrm{S}}^{\mathrm{LO}+\mathrm{NLO}}\right|_{\text {const. }} \rightarrow G_{\mathrm{S}}^{\mathrm{LO}+\mathrm{NLO}}\right|_{\text {const. }} \times R .
$$

The result is shown in fig. 3(left), and we find no substantial improvement.

The second test concerns the threshold region. Thermal corrections modify the threshold location by a well-known NLO correction [10],

$$
m^{2} \rightarrow m^{2}+g^{2} T^{2} C_{\mathrm{F}} / 6,
$$

which tends to move the threshold to larger frequencies. For a large quark mass, however, the effect is small: $\delta \omega_{\text {threshold }}=g^{2} T^{2} C_{\mathrm{F}} /(12 m)$. There is an effect of opposite sign originating from a Debye-screening induced correction to a heavy quark mass, $\delta \omega_{\text {threshold }}=-g^{2} C_{\mathrm{F}} m_{\mathrm{D}} /(4 \pi)[11]$. However, apart from these thermal corrections, there is also an important zero-temperature effect: whereas accounting for the small- $\tau$ behaviour of $G_{\mathrm{S}}$ requires the use of the $\overline{\mathrm{MS}}$ scheme or a similar running mass [2], it is known that threshold features are better described by a pole-type mass (cf. e.g. ref. [12]). Like in fig. 1 we denote the pole mass by $M$.

In order to probe these effects, we have considered a shift of the threshold location by multiplying, in effects originating from quark propagators, the quark mass by a factor $Q$. However the 

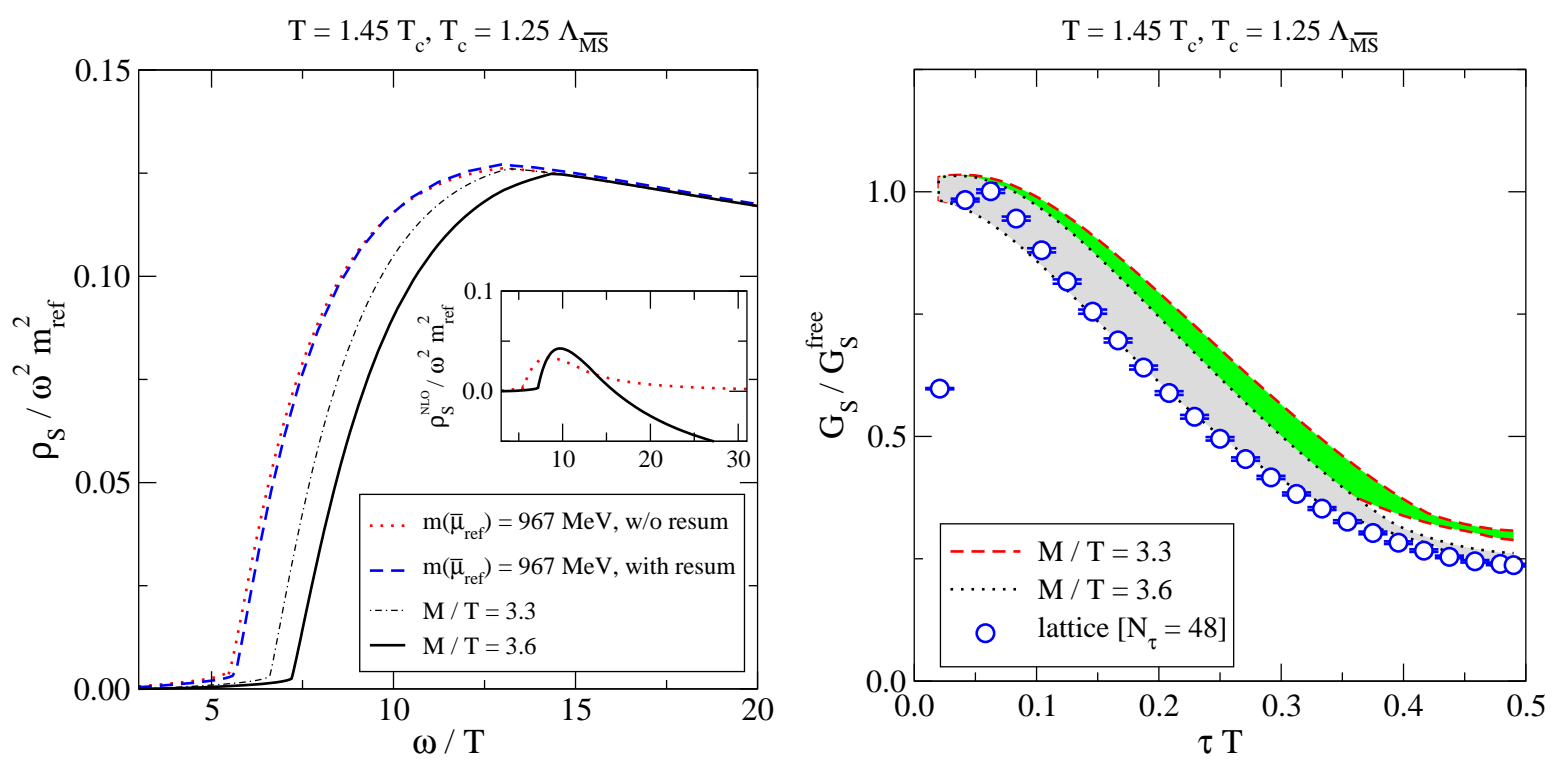

Figure 4: Left: The LO+NLO scalar channel spectral function around the threshold, with and without mass resummation in the $\overline{\mathrm{MS}}$ scheme (cf. eq. (4.2)), and with mass resummation in the pole mass scheme. The inset shows the NLO parts and illustrates the non-convergence of the pole mass result at large $\omega$. Right: The imaginary-time correlator, from a rescaled pole mass result at $\omega<4 M$ and $\overline{\mathrm{MS}}$ result at $\omega>4 M$, compared with lattice data [3]. The remaining discrepancy is probably due to the non-relativistic approximation.

overall multiplicative factor $m^{2}$ originating from eq. (2.2) is left unchanged. With a particular scale choice [2], the location of the zero-temperature threshold is thus given by the solution of

$$
\omega_{\text {threshold }}=2 m\left(\bar{\mu}=\omega_{\text {threshold }} e^{-17 / 12}\right) \times Q .
$$

The result is shown in fig. 3(right); the discrepancy is considerably reduced for $Q>1$. If we recall from fig. 2 that the non-relativistic approximation overestimates the true answer at these quark masses, the optimal value might be $Q \simeq 1.2$ or so. Remarkably, this is quite close to the ratio of the pole and $\overline{\mathrm{MS}}$ masses for the parameter values used in ref. [3],

$$
\frac{M}{m_{c}\left(m_{c}\right)}=1+\frac{4 g^{2}\left(m_{c}\right) C_{\mathrm{F}}}{(4 \pi)^{2}}+\mathscr{O}\left(g^{4}\right) \approx \frac{1.3 \mathrm{GeV}}{1.1 \mathrm{GeV}} \approx 1.2 .
$$

As a crosscheck, we have employed the same pole masses $M / T \approx 3.3,3.6$ as in fig. 1(left) for treating the scalar channel threshold region. More concretely, we have considered the NLO spectral function as given in ref. [7], which was indeed in the pole mass scheme, as well as the corresponding $\overline{\mathrm{MS}}$ scheme one, with or without thermal mass resummation. ${ }^{1}$ The pole mass result is not reliable at large $\omega$, because the NLO correction overtakes the LO term and the perturbative series breaks down, cf. the inset in fig. 4(left). We normalize the pole mass result such that it agrees with the unresummed $\overline{\mathrm{MS}}$ result at $\omega=4 M$; above this, the $\overline{\mathrm{MS}}$ result is used, cf. fig. 4(left). The resulting imaginary-time correlators are illustrated in fig. 4(right), together with a comparison with lattice data. The agreement is much better than in fig. 1(right), and remarkably good considering that there are errors related to the non-relativistic approximation as visible in fig. 2.

\footnotetext{
${ }^{1}$ The $\overline{\mathrm{MS}}$ scheme result is obtained from the expressions of ref. [7] by setting $M \rightarrow m(\bar{\mu})$ and $\delta \rightarrow-\ln \left[\bar{\mu}^{2} / m^{2}(\bar{\mu})\right]-$ 4/3. Thermal mass resummation can be removed by changing $+4 k^{2} \rightarrow-2 k^{2}$ in eq. (C.11).
} 


\section{Scalar channel spectral function in the bottom quark case}

In the bottom quark case there is no doubt about the validity of the non-relativistic approximation. This permits the use of special effective theories, such as Heavy Quark Effective Theory for addressing the transport region [14] and Non-Relativistic QCD for addressing the threshold region [15]. One issue of phenomenological controversy is that whereas there is certainly no resonance peak in the scalar spectral function for $M<2 \mathrm{GeV}$, for the bottom case $M \simeq 4.5 \mathrm{GeV}$ a small $S$-channel contribution has been suggested to appear in the dominantly $P$-channel scalar correlator [13]. This induces a peak to the corresponding spectral function. It will be interesting to see whether a peak can be resolved from data $[16,17]$ with refined spectral analysis tools [18].

\section{Conclusions}

The study of charm quark correlators in hot QCD may soon enter a mature phase. On the lattice side a continuum limit remains to be taken, but the lattices used are already in a scaling regime, at least in the quenched case. On the continuum side, full NLO spectral functions need to be computed, going beyond the present non-relativistic approximation. Once these steps have been taken, the short- $\tau$ regimes of the two sides should match, and a non-divergent difference from the large- $\tau$ regime could be subjected to a spectral analysis of genuine non-perturbative effects.

This work was partly supported SNF under the grants 200021-140234 and PZ00P2-142524.

\section{References}

[1] Y. Burnier and M. Laine, JHEP 11 (2012) 086 [1210.1064].

[2] Y. Burnier and M. Laine, 1309.1573.

[3] H.-T. Ding et al, Phys. Rev. D 86 (2012) 014509 [1204.4945].

[4] J. Beringer et al. [Particle Data Group Collaboration], Phys. Rev. D 86 (2012) 010001.

[5] D.J. Broadhurst et al, Z. Phys. C 60 (1993) 287 [hep-ph/9304303].

[6] K.G. Chetyrkin, J.H. Kühn and M. Steinhauser, Nucl. Phys. B 505 (1997) 40 [hep-ph/9705254].

[7] Y. Burnier, M. Laine and M. Vepsäläinen, JHEP 02 (2009) 008 [0812.2105].

[8] M. Laine, 1310.0164.

[9] D. Bödeker and M. Laine, JHEP 07 (2012) 130 [1205.4987].

[10] J.F. Donoghue et al, Annals Phys. 164 (1985) 233 [Erratum-ibid. 172 (1986) 483].

[11] R.D. Pisarski, Phys. Rev. Lett. 63 (1989) 1129.

[12] M. Beneke, hep-ph/9911490.

[13] Y. Burnier, M. Laine and M. Vepsäläinen, JHEP 01 (2008) 043 [0711.1743].

[14] S. Caron-Huot, M. Laine and G.D. Moore, JHEP 04 (2009) 053 [0901.1195].

[15] G. Aarts et al, J. Phys. Conf. Ser. 432 (2013) 012014 [1212.4368].

[16] S. Kim, P. Petreczky and A. Rothkopf, PoS LATTICE 2013 (2013) 169.

[17] G. Aarts et al, 1310.5467.

[18] Y. Burnier and A. Rothkopf, 1307.6106. 